\title{
Study and Design of Home Network Control System Based on Electronic Engineering
}

\author{
Wu Yongchong \\ Jiangxi University of Technology,Continuing Education Institute
}

\begin{abstract}
With the continuous improvement and development of technology, electronic engineering develops into automation gradually. With the increasing demand for electronic technology, electronic technology has gradually become the most important project of network engineering, and wireless home network control system has been gradually into the modern family, as a new thing in the field of communication and electrical engineering. This paper compares the current international popular home network control systems and proposes a new scheme based on the wireless chip systems, that is, the home network control system combining wireless communication technology, embedded technology, GUI technology and computer network technology. The paper introduces the key technology, hardware and software design and examples, and finally discusses the future direction of development.
\end{abstract}

Key words- Electronic engineering; Embedded type; Computer network system; GUI

\section{I.INTRODUCTION}

At present, the development of China's electronic engineering becomes relatively active, and electronic engineering is very important for the promotion of employment, stimulating economic growth, adjusting the industrial structure, changing the mode of economic development and the maintenance of national security. Under the support of constantly updated information technology, the application in modern technology of electronic engineering correspondingly becomes larger.

With the improvement of living standards, accelerated pace of work, and improving living standards, people increasingly want to be able to achieve the centralized intelligent management of household equipment. The establishment of a digital, networked home can save the labor cost, timely access to the information and exchange more information with the outside world, so that people can be free from the heavy housework. Therefore, the establishment of the home network control system becomes a very important and urgent need. Home network control system is the product of combining computer, communication, control and network and other related technology with the traditional residence, that is the combination of residence of internal devices and application system in order to achieve a more comfortable and safe, economical and convenient home. The definition and function expression of home networking are various, and the main current functions still include household appliances control, security control, resource control, medical control. Through home network system, users can fully and effectively manage and control a variety of home equipment with different characteristics, and improve the living environment comfort, and freed from the heavy housework activities. It's also has a significant practical meaning in security and energy control and other aspects.

The paper includes the description of the development and status of home network control system, and analysis and comments for the existing research results; analysis of the development trends of connection technology using home network control system; analysis and comparison for several wireless home network control systems currently used; Finally, the paper proposes the overall structure based on the home network control system on the wireless chip, and clarifies the technical advantages of the program.

\section{II.THE CONNOTATION OF ELECTRONIC ENGINEERING}

Due to the continuous development of the Internet, network technology also made great progress with this while promoting electronic technology becoming an independent industry. The $21 \mathrm{st}$ century is the times when Internet technology promotes the economic development with electronic engineering and the appliance. In order to better promote the development of electronic engineering, better deal with international challenges as well as promote the development of electronic technology, we must strengthen the exploration and research of electronic engineering.

As a discipline, electronic engineering is a science taking use of computer technology and network technology to process and control the electronic information, mainly related to application development of electronic equipment and systems, and related contents of information processing. Now that the products in people's lives are increasingly dependent on electronic technology, so it affects all aspects of people. Through in-depth study of related contents, our new product development process can be significantly promoted, enabling us to develop more new electronic engineering industries.

As the first century of information age, the 21 st century is a succession century for the development of science to promote the development of the industry and make electronic engineering enter a new era of development. In many places, the electronic technology is a good new type of industry, which promotes the development of industry of local economy and the improvement of China's comprehensive national strength on the side. Now, China is in the rapid development of the national economy, we are deepening 
the development of electronic technology industry, and provide related support. This provides an opportunity for the development of electronic engineering, and improves the overall strength of the development of electronic engineering.

\section{III.IMPLEMENTATION TECHNIQUES OF THE EXISTING}

\section{WIRELESS HOME NETWORK CONTROL SYSTEM}

The carrier of bluetooth adopts the universal $2.4 \mathrm{GHz}$ ISM band, ie industrial, scientific and medical band. The center frequency of bluetooth is $2.45 \mathrm{GHz}$ that can get 79 available channels with $1 \mathrm{MHz}$ bandwidth. Bluetooth uses GFSK modulation technology with transmission rate of $1 \mathrm{Mb} / \mathrm{s}$, and the real effective rate up to $721 \mathrm{~Kb} / \mathrm{s}$, transmission distance generally for $10 \mathrm{~m}$. Communication protocol often uses Time Division Multiple Access (TDMA) protocol that various types of data and voice devices following the Bluetooth protocol can wirelessly access to the public network (eg Internet, Intranet). Bluetooth system is based on PPP(Point-to-point protocol) connection, using wireless way to connect several bluetooth equipment into a topology structure microgrid (Piocnt) of a master-slave device. Each Piocnet Bluetooth equipment consists of a master and several slave devices, and the master device is responsible for the operation of the communication protocol. Addressing equipment within a piconet device can reach to 8 . Figure 1 shows a topology map of master-slave device in 1 piconet. In addition, Bluetooth system also supports the distributed network consisted by point-to-multipoint communication, namely a master device can be the salve unit of other Piconet, and each slave device can also be the master unit of other Piocnet.

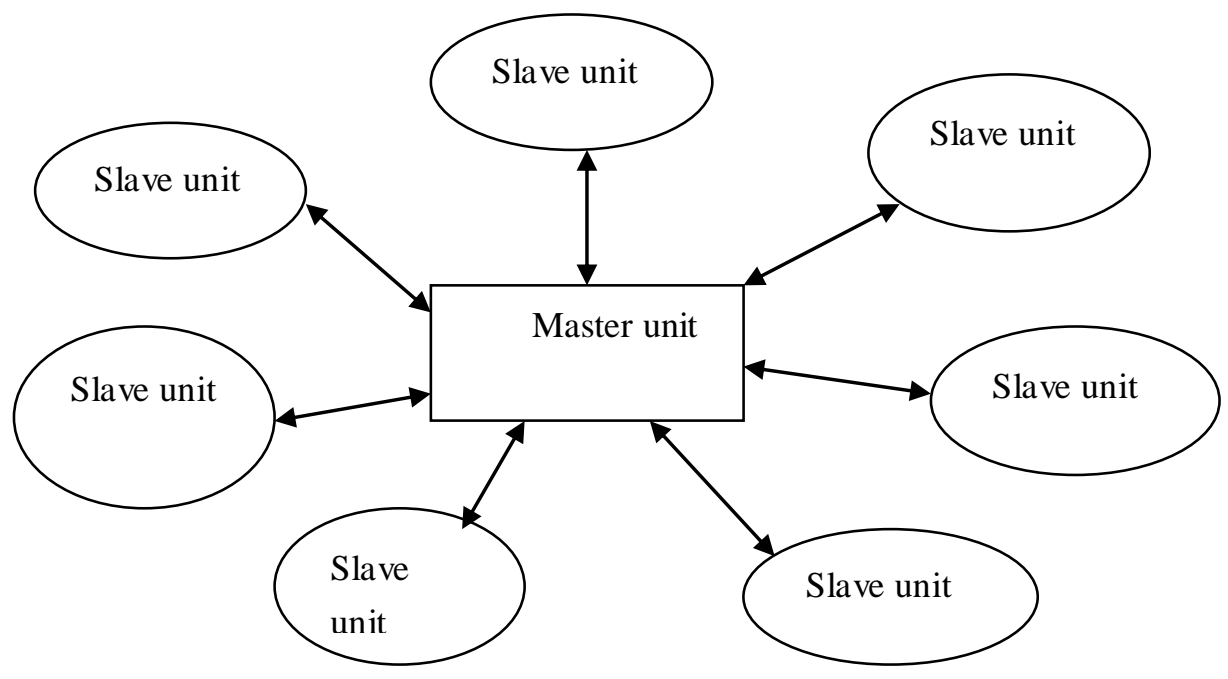

Figure 1. Topology structure of bluetooth primary and slave equipment

The disadvantages of bluetooth technology:

The transmission distance of bluetooth is usually less than $10 \mathrm{~m}$, which is a disadvantage that is difficult to overcome in a bigger residence. So it is difficult to build up home wireless communication network.

\section{IV.DESIGN OF EMBEDDED CENTRALCONTROL UNIT}

Embedded central controller includes an embedded processor, a coprocessor, the external control circuitry, memory (including read-only, read-write), and peripheral expansion devices. In the selection of a variety of hardware chips, features, power consumption, size, cost, reliability, electromagnetic compatibility, ambient temperature and so on should be taken into account.

The overall structure of embedded core board hardware design is shown as Fig .2. 


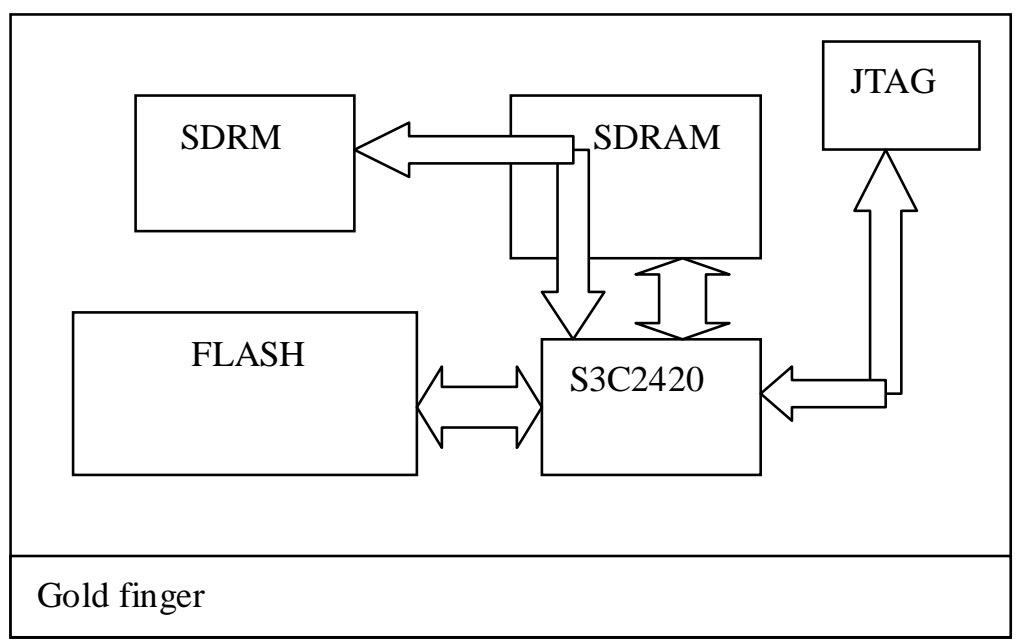

Figure 2. Structure chart of core board

Samsung 53C240 processor, 64M50 and 16M FLASH are integrated into the core-board. 8 laminates technology is adopted with EMC test, assembly welding and open bus resources opening and almost all pins of 53C2410 are extracted through the expansion socket (Goldfinger). In Figure 2, there is no welding on the chip in the finished goods figure. The entire core board includes our own design, wiring, welding (except BGA packaged sc2410) and debugging. We have now completed the debugging of the first edition.

\section{V.CONCLUSION}

Embedded central controller also integrates the digital signal processor TMS32OVC5509 (DSP) manufactured by $\mathrm{TI}$ on the back plane. It uses the modified Harvard architecture, and the operating rate can reach 100MPIS. Samsung S3C241O processor controls the digital signal processor (DSP) through EHPI. The signal processing based on wavelet transformation is completed by TMS320VC5509 digital signal processor (DSP), with the powerful digital signal processing capability of digital signal processor and our existing self-developed algorithm that can process a lot of signals in a short time. We have also recently prepared using FPGA for processing digital signals, to realize hardware compression, improve efficiency and reduce costs.

In this paper, home network control system is the object for the study, based on the wireless chip system, and a practical prototype is also developed.

\section{REFERENCE}

[1] Shen, Hongrui, et al. "Electronic ticket system based on embedded and RFID technology." Microcomputer \& Its Applications (2011)
[2] Zhu M J, Pan Z X, Luo H. The Experience Teaching Exploration of Embedded System on Electronic and Information Engineering $[\mathrm{J}]$. Journal of Electrical \& Electronic Education, 2012.

[3] Xia Q B, Ya-Ping M A, Yi L U. Research on the international standard electronic chart display system based on embedded system[J]. Science of Surveying \& Mapping, 2008.

[4] Gao F, LI-Meng. Exploration on the Construction of Electric and Electronic Engineering Training Center[J]. Research \& Exploration in Laboratory, 2006.

[5] Alder C. Software engineering education in an electronic engineering degree[J]. Software Engineering Journal, 1989, 4(4): 191 - 199.

[6] Zhang, Jun He, and H. V. College. "Discussion Mechanical and Electronic Engineering and Artificial Intelligence." Equipment Manufacturing Technology (2014).

[7] Friesel, A. "Retaining electronic engineering students by project-and team-work from the first semester." Education Engineering (EDUCON), 2010 IEEE IEEE, 2010:1327-1333.

[8] Al-Zoubi A Y, Nsour J, Jeschke S, et al. An Electronic Engineering Mobile Remote Laboratory[J]. Automation Communication \& Cybernetics in Science \& Engineering, 2009:289-299.

[9] Wang, Liting, X. Ding, and C. Fang. "Department of Electronic Engineering, Tsinghua University, Beijing 100084, China." Tsinghua Science \& Technology 14.6(2009):685-690.

[10] Yao, Xiao Qun. "The Study on Teaching Approaches in Mechanical and Electronic Engineering." Journal of Guangdong Communications Polytechnic (2007).

[11] Chang, Liang Te. "Using Revised DACUM and Survey To Determine Electronic Engineering Technology Curriculum.." Curriculum Development (1996): 16.

[12] Greene, J. R. "Experience-based learning in electrical and electronic engineering: An effective and affordable approach." AFRICON '92 Proceedings., 3rd AFRICON Conference IEEE, 1992:10-13. 